TAO, Vol. 16, No. 3, 547-561, August 2005

\title{
Inverse-Ray Imaging of a 3D Homogeneous Layer Based on Common-Shot Gathers of Oblique Profiles
}

\author{
Tan-Kin Wang ${ }^{1, *}$ and Kai-Ming Chang ${ }^{1}$
}

(Manuscript received 29 July 2004, in final form 23 May 2005)

\begin{abstract}
Imaging of 3D structural interfaces through reflected rays shooting from common-shot gathers is presented in this paper. First, by fitting the reflected arrivals picked from common-shot gathers, we calculate apparent dips and the shortest distances between sources and reflectors along two profiles. Then, based on the geometry of the profiles and a planar reflector, a unit normal vector of the reflector is determined from the apparent dips and the azimuths of two oblique profiles. A special case, when apparent dips are zero along two parallel profiles, for determining the reflector normal is also investigated. We propose three criteria to ensure the selected travel-times along two profiles resulting from the same planar reflector. These are that firstly, the same shortest distance from sources to the reflector is utilized; and secondly, we want to ensure the same normal of the reflector, and finally, the same ray distance.

Prestack inverse-rays developed in this paper are applied to image the bathymetry of the Hoping Basin in the southernmost Ryukyu subduction zone and the fourth layer of the SEG/EAEG over-thrust model. Based on common-shot gathers along seven oblique profiles in the Hoping Basin, most of the reflection points are well imaged through inverse rays except when variation of the interface depth exceeds $300 \mathrm{~m}$ across profiles with spacing greater than about $20 \mathrm{~km}$. Inverse-ray imaging of the over-thrust model also provides good agreement to its fourth interface except that imaging errors of about $1 \mathrm{~km}$ in depth are found near the thrust faults.

Inverse-ray imaging of 3D structures from 2D multi-channel seismic profiles is demonstrated if a pseudo-3D structure (a planar reflector) exists

\footnotetext{
${ }^{1}$ Institute of Applied Geosciences, National Taiwan Ocean University, Keelung, Taiwan, ROC

* Corresponding author address: Prof. Tan K. Wang, Institute of Applied Geosciences, National Taiwan Ocean University, Keelung, Taiwan, ROC; E-mail: tkwang@mail.ntou.edu.tw
} 


\begin{abstract}
between profiles or if at least two profiles are within a Fresnel zone. Although this technique deals with a single-layered problem currently, it is fundamentally important when we extend it to image inhomogeneous multilayered media.
\end{abstract}

\author{
(Key words: Apparent dip, Migration, Multi-channel seismic, Ray geometry, \\ Reflector, Travel time)
}

\title{
1. INTRODUCTION
}

Prestack depth migration has been widely used in the multi-channel seismic (MCS) data processing to image 3D complex structures such as faults, salt domes and gas sags (Gray et al. 2001). However, the main drawbacks of the prestack depth migration are its computation time for large data sets and the sensitivity of the data quality. Recently, ray-based techniques (Operto et al. 2000; Hua and McMechan 2003) were incorporated into the prestack depth migration for enhancing computation speed, but its applications in migration velocity analysis (MVA) and real-time monitoring are still limited (Donihoo et al. 2001).

On the other hand, geometrical migration, for which only travel times are considered, has been developed since the pioneering work of poststack migration. By considering travel times and their gradients from stacked sections along orthogonal profiles, Sattlegger (1969) and Kleyn (1977) first proposed the "map migration" based on 3D inverse rays. A 3D inverse ray in their study was traced from a receiver, with a shooting direction calculated from travel-time gradients along orthogonal profiles, and traveled through the upper layers with one-way time until it arrived at the image point. May and Covey (1981) used inverse reflected and diffracted rays to image complex faults. They suggested that the error in inverse-ray imaging results mainly from the travel-time gradient which may accumulate large errors in imaging lower layers. Since model velocity is required before implementing inverse ray tracing, 3D inverseray imaging was also emphasized for velocity determination (Gjoystdal and Ursin 1981; Maber and Hadley 1986; Reilly 1991). Recently, Wang (2005a) developed a 3D inverse-ray imaging technique based on common-midpoint (CMP) triangulation of oblique and sparse profiles and incorporated MVA for enhancing the velocity determination.

Similar to the development from the poststack to the prestack depth migration, inverse rays have been applied to prestack imaging in the past decade. Wang (2002) computed the reflection points at a $2 \mathrm{D}$ irregular interface from the ray geometry and the elliptical envelope. Ray geometry, involving a virtual source and a virtual receiver with respect to a planar reflector, provides the shooting direction of an inverse ray whereas a tangent plane of elliptical envelopes leads to a reflector. By considering the least-square travel-time fits, the method of elliptical envelopes was also extended to determine the strike and the true dip of 3D reflectors (Pivot 1997). In addition to the reflection tomography, a so-called stereo-tomography (Billette and Lambare 1998; Billette et al. 2003) was proposed by including travel-time slopes of common-shot and common-receiver gathers to calculate the prestack inverse rays propagating in $2 \mathrm{D}$ multi-layered media. With the aid of the $2 \mathrm{D}$ prestack inverse rays and by assuming the 


\section{Tan Kin Wang \& Kai Ming Chang}

fixed velocity of the upper layer, an exact velocity analysis of the lowest layer was also presented recently (Wang 2005b).

The aim of this study is to fully use the existing 2D MCS profiles for constructing 3D structures since a 3D seismic survey is expensive. In this paper, we develop a prestack inverseray imaging of a 3D homogeneous layer based on inverse rays propagating along MCS profiles. Although the target for prestack imaging of a 3D homogeneous layer is similar to Pivot's work (1997), this technique is more efficient for handling MCS data recorded along oblique profiles. Furthermore, by considering the travel-time gradients of MCS data, this method can be extended for imaging 3D inhomogeneous multi-layered media.

In the following, we determine the side effect of inverse rays perpendicular to the profiles from at least two common-shot gathers recorded along oblique profiles. Based on the ray geometry of apparent dips along oblique profiles, a unit normal vector of a planar reflector and the reflection points are obtained (Chang 2004). We further propose three criteria for selecting the proper travel-time sets from all common-shot gathers. Finally, the prestack inverse rays are applied to image the bathymetry of a forearc basin, in the southernmost Ryukyu subduction zone where MCS lines are dense, and a complex model of SEG/EAEG over-thrust.

\section{THEORY}

Inverse rays propagating in 3D can be determined based on the ray geometry from at least two non-parallel profiles. By considering the least-square travel-time fitting of a commonshot gather along a profile, Wang (2002) derived the velocity $(V)$ of a homogeneous layer, the apparent dip $\left(\alpha_{i}-\pi / 2\right)$ of a planar reflector, and the shortest distance $\left(h_{i}\right)$ between the source $\left(S_{i}\right)$ and the reflector (Fig. 1). Therefore, a unit normal vector of a 3D planar reflector can be calculated from apparent dips of the reflector based on two common-shot gathers along two oblique profiles (Appendix A). However, the question arises as to how we can properly select two common-shot gathers in order to calculate an accurate normal of a planar reflector. In this paper, we present three criteria for selection of two common-shot gathers to determine the reflector normal and the reflection points.

\subsection{Criteria for Incorporating Travel-Time Sets}

For determining a reflector normal, travel-time sets of two common-shot gathers along two profiles are selected from reflected arrivals of all 2D MCS profiles. We propose the following criteria to ensure the selected travel-times result from the same planar reflector.

\subsubsection{The Shortest Distance between the Source and the Reflector}

Let the travel-time sets be selected from two common-shot gathers along oblique profiles (or the apparent dips are zero along parallel profiles) for which the shortest distances $\left(h_{i}\right.$ or $\left.h_{j}\right)$ between the sources $\left(S_{i}\right.$ or $\left.S_{j}\right)$ and the reflector can be determined from fitting of the travel-time sets along profiles (Wang 2002). In particular, a reflection point $\left(S_{j}^{*}\right)$ of a ray emitting from the source $\left(S_{j}\right)$ and reflected back to the same source (Fig. 1) can be written as: 


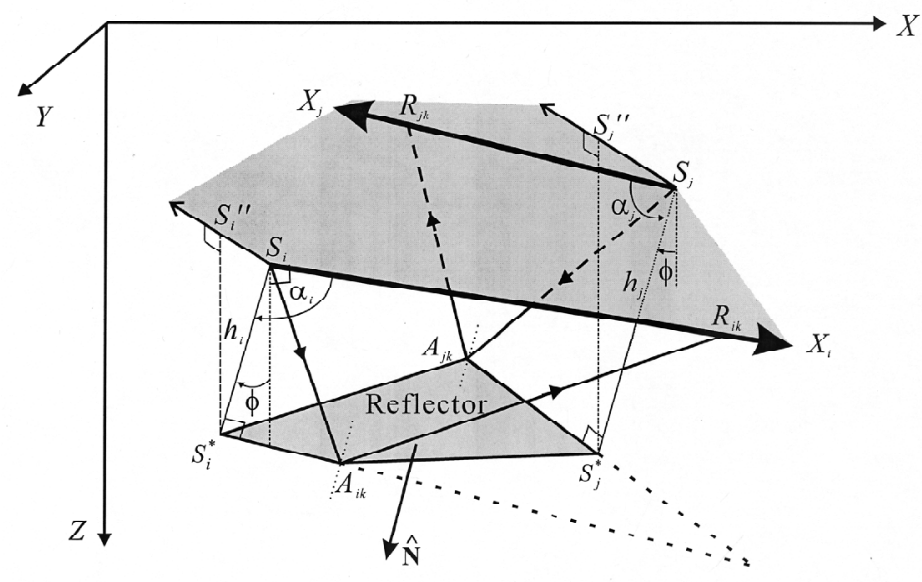

Fig. 1. Geometry of reflected rays shooting from sources $\left(S_{i}\right.$ and $\left.S_{j}\right)$ at the surface (XY plane), reflecting at a planar reflector $\left(S_{i}^{*}, A_{i k}, S_{j}{ }^{*}\right.$, and $\left.A_{j k}\right)$, and arriving at receivers $\left(R_{i k}\right.$ and $\left.R_{j k}\right)$ along two oblique profiles $\left(X_{i}\right.$ and $\left.X_{j}\right) . h_{i}$ and $h_{j}$ are the shortest distances from sources $S_{i}$ and $S_{j}$ to the reflector, respectively. Direction angles of $\overrightarrow{S_{i} S_{i}^{*}}\left(\right.$ or $\left.\overrightarrow{S_{j} S_{j}^{*}}\right)$ with respect to the profile and $Z$ axis are denoted by $\alpha_{i}\left(\right.$ or $\left.\alpha_{j}\right)$ and $\phi$, respectively. Projection of $\overrightarrow{S_{i} S_{i}^{*}}\left(\right.$ or $\left.\overrightarrow{S_{j} S_{j}^{*}}\right)$ onto the surface, $\overrightarrow{S_{i} S_{i}^{\prime \prime}}$ (or $\overrightarrow{S_{j} S_{j}^{\prime \prime}}$ ), is along the dip line of the reflector.

$$
S_{j}^{*}:\left(S_{j x}+h_{j} N_{x}, S_{j y}+h_{j} N_{y}, h_{j} N_{z}\right)
$$

If the travel-time sets are associated with the same reflector, then projection of the vector $\overrightarrow{S_{i} S_{j}^{*}}$ onto the reflector normal should be equal to the shortest distance $h_{i}$, i.e.,

$$
h_{i}=\overrightarrow{S_{i} S_{j}^{*}} \cdot \hat{\mathbf{N}}
$$

To substitute equation (1) into equation (2), we derive the first criterion

$$
h_{i}-h_{j}=\overrightarrow{S_{i} S_{j}} \cdot \hat{\mathbf{N}}
$$

of properly incorporating the travel-time sets for which the shortest distances $\left(h_{i}\right.$ and $\left.h_{j}\right)$ between the sources $\left(S_{i}\right.$ and $\left.S_{j}\right)$ and the unit normal $(\hat{\mathbf{N}})$ can be determined. 
Tan Kin Wang \& Kai Ming Chang

\subsubsection{The normal Vector of the Reflector}

If an apparent dip along a profile is not zero (or $\alpha_{i} \neq \pi / 2$ in Figs. 2a, b), then, by considering the side effect perpendicular to the profile, the reflector is a tangent plane of a circular cone rotated about the profile and with a vertex:

$$
B_{i}:\left(S_{i x}+\frac{h_{i}}{\cos \alpha_{i}} \cos \theta_{i}^{*}, S_{i y}+\frac{h_{i}}{\cos \alpha_{i}} \sin \theta_{i}^{*}, 0\right),
$$

at the surface (Fig. 2a, b). The vector $\overrightarrow{B_{i} S_{i}^{*}}$ and $\overrightarrow{S_{i}^{*} S_{j}^{*}}$ lie on the reflector and can be written as:

$$
\overrightarrow{B_{i} S_{i}^{*}}=h_{i} \hat{\mathbf{N}}-\frac{h_{i}}{\cos \alpha_{i}} \hat{X}_{i} \text { and } \overrightarrow{S_{i}^{*} S_{j}^{*}}=\overrightarrow{S_{i} S_{j}}+\left(h_{j}-h_{i}\right) \hat{\mathbf{N}}
$$

with the aid of equations (1) and (4). Therefore, the cross product of two vector $\overrightarrow{B_{i} S_{i}^{*}}$ and $\overrightarrow{S_{i}^{*} S_{j}^{*}}$ should be checked to see whether it is parallel to the reflector normal $(\hat{\mathbf{N}})$.

Alternatively, if an apparent dip along a profile is zero (or $\alpha_{j}=\pi / 2$ ), then the reflector is a tangent plane of a circular cylinder rotated about the profile and with a radius of $h_{j}$ (Figs. 2b, c). In this case, the vector $\hat{X}_{j}$ along the profile lies also at the reflector. Therefore, the cross product of vectors $\hat{X}_{j}$ and $\overrightarrow{S_{i}^{*} S_{j}^{*}}$ should be parallel to the reflector normal. Special cases of the apparent dips about zero along two parallel profiles (Fig. A2) and along two oblique profiles (Fig. 2c) should also be investigated. According to equations (A4) and (A7), the true dip of the reflector for the later case is zero whereas the reflector of the former case is not necessarily horizontal. It can be also readily shown that the criteria introduced in Section 2.1 apply for both special cases.

\subsection{Computation of Reflection Points}

If a unit normal vector of a reflector $(\hat{\mathbf{N}})$, computed from equation (A4) or (A7) by considering two common-shot gathers along two profiles satisfies the criteria introduced in Section 2.1, we can determine the reflection points on the planar reflector. Let reflected rays be shot from a common source $\left(S_{i}\right)$, be reflected at reflection points $\left(A_{i j}\right)$, and arrive at multichannel receivers $\left(R_{i j}\right)$. According to the ray geometry in Fig. 3, the shortest distances between the receivers and the reflector are:

$$
H_{i j}=h_{i}+\overline{S_{i} R_{i j}} \sin \left(\alpha_{i}-\frac{\pi}{2}\right)=h_{i}-\overline{S_{i} R_{i j}} \cos \alpha_{i}
$$


(a)

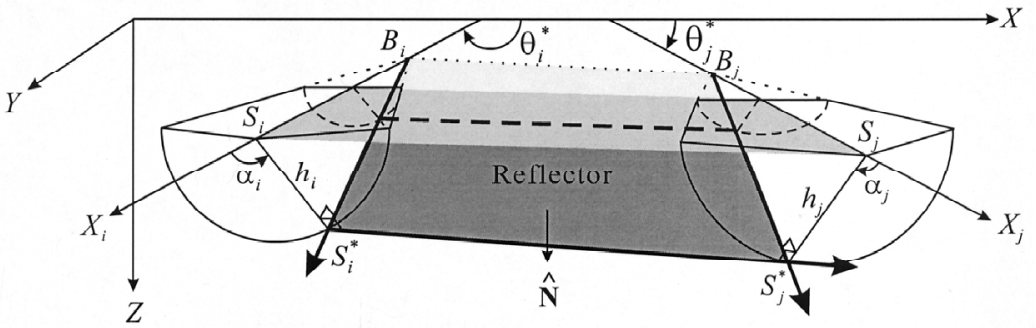

(b)

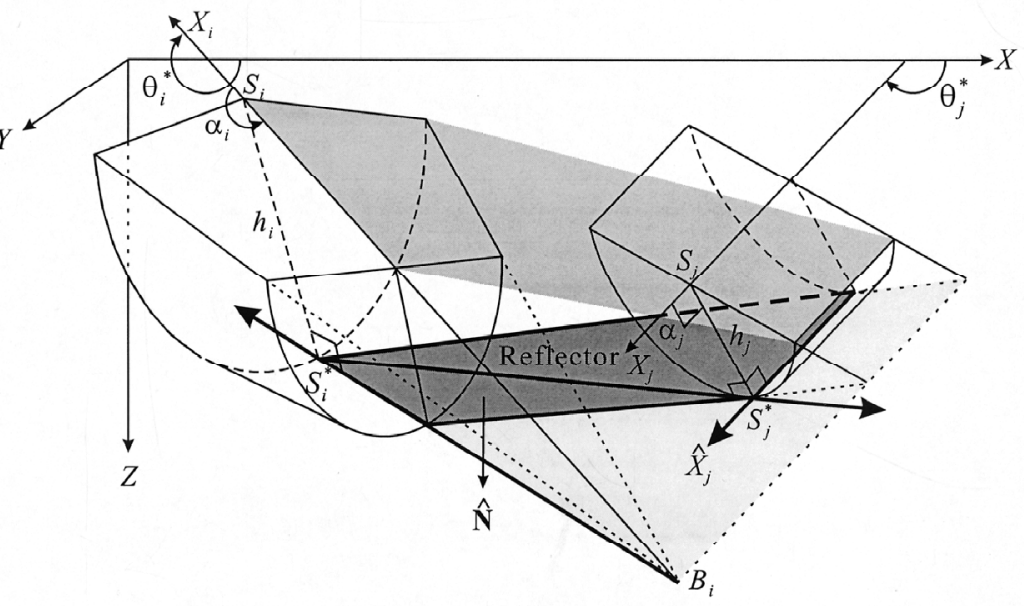

(c)

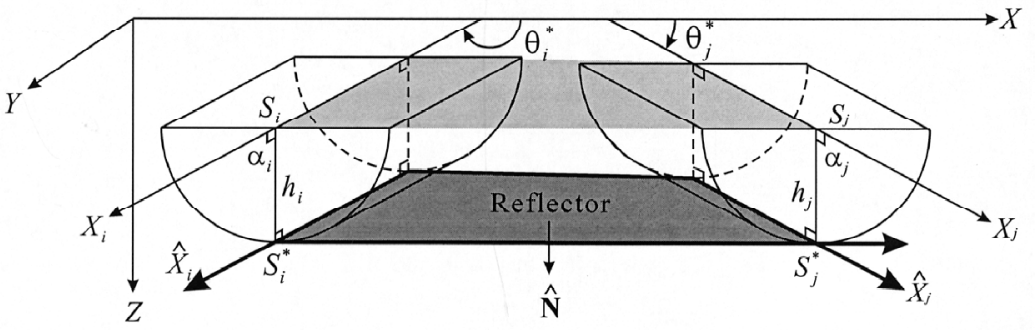

Fig. 2. Planar reflectors imaged from tangent planes of (a) two circularly conical envelopes when $\alpha_{i}, \alpha_{j} \neq \pi / 2$, (b) a circularly conical envelope when $\alpha_{i} \neq \pi / 2$ and a circularly cylindrical envelope when $\alpha_{j}=\pi / 2$, and (c) two circularly cylindrical envelopes when $\alpha_{i}, \alpha_{j}=\pi / 2$ along oblique profiles $X_{i}$ and $X_{j} . B_{i}$ and $B_{j}$ are vertices of circularly conical envelopes along profiles $X_{i}$ and $X_{j}$, respectively. Vectors $\overrightarrow{S_{i} S_{j}^{*}}, \overrightarrow{B_{i} S_{i}^{*}}, \overrightarrow{B_{j} S_{j}^{*}}$, $\hat{X}_{i}$, and $\hat{X}_{j}$ lie on the planar reflectors. 


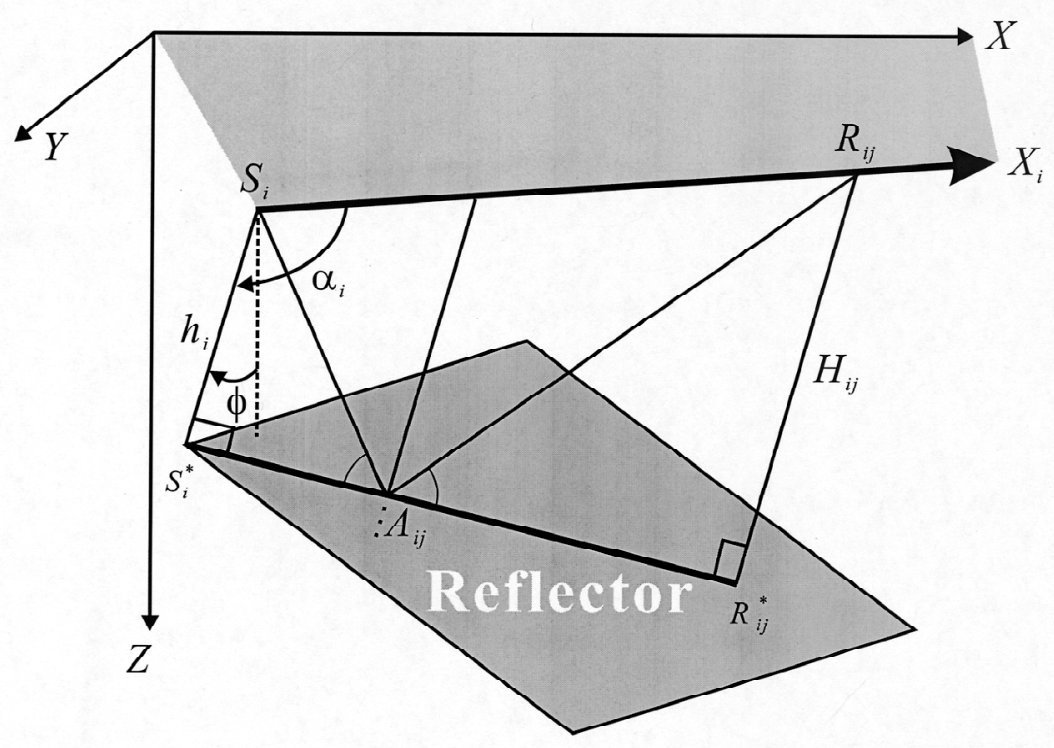

Fig. 3. Reflection point $\left(A_{i j}\right)$ determined from a ray shooting at a source $\left(S_{i}\right)$ and arriving at a receiver $\left(R_{i j}\right) . h_{i}$ and $H_{i j}$ are the shortest distances, respectively, from the source and from the receiver to the reflector.

Since $\Delta S_{i} S_{i}^{*} A_{i j}$ and $\Delta R_{i j} R_{i j}^{*} A_{i j}$ are similar triangles, the reflection points are obtained.

$$
A_{i j k}=S_{i k}^{*}+\frac{h_{i}}{h_{i}+H_{i j}}\left(R_{i j k}^{*}-S_{i k}^{*}\right), k=x, y, z \text {, }
$$

where projection of the source $\left(S_{i}\right)$ and the receivers $\left(R_{i j}\right)$ along the reflector normal and onto the reflector are:

$$
S_{i k}^{*}=S_{i k}+h_{i} N_{k}, R_{i j k}^{*}=R_{i j k}+H_{i j} N_{k}, k=x, y, z .
$$

Finally, we can also check whether the ray distance and the layer velocity are consistent with the reflected travel-times:

$$
T_{i j}=\frac{\overline{S_{i} A_{i j}}+\overline{R_{i j} A_{i j}}}{V_{i}} .
$$




\section{RESULTS}

\subsection{Hoping Basin}

A forearc basin, Hoping Basin, is located near the Ryukyu Arc, Luzon Arc and Yaeyama Accretionary Prism in the southernmost Ryukyu subduction zone where the plate motion and earthquakes are active (Fig. 4). Subduction and arc-continent collision in the Ryukyu forearc region have resulted in an old Suao Basin beneath the Hoping Basin (Lallemand et al. 1997) and warping of the arc basement east of the Hoping Basin (Wang et al. 2004). Furthermore, on 31 March 2002, an earthquake with a magnitude of 6.8 and a focal depth of $9.6 \mathrm{~km}$ beneath the Hoping Basin resulted in strong damage in Taipei at an epicentral distance of about $100 \mathrm{~km}$. Based on migration velocity analysis and horizon picks over MCS stacked sections, four major sedimentary layers of the Hoping Basin are imaged through 3D inverse reflected rays (Wang 2005a). In this paper, we use reflected arrivals of the common-shot gathers along seven MCS profiles in the Hoping Basin and apply the inverse rays to image the bathymetry of the basin.

Reflected rays are traced on the high-resolution bathymetry of the Hoping Basin (Fig. 5a). For inverse-ray imaging along seven MCS profiles, there are 450 shots with a shot interval of $1.25 \mathrm{~km}$ and a receiver interval of $0.5 \mathrm{~km}$ that are less dense than the real data set. According to the smoothness and continuity of the travel-time curves resulting from the same planar reflector, any two travel-time sets of common-shot gathers along oblique profiles are selected for determining the reflector normal from equation (A4). Criteria of the shortest distance between the source and the reflector, the reflector normal and the ray distance introduced in Sections 2.1 and 2.2 are considered to delete the improper selection of the travel-time sets.

Since the basin bathymetry (width, length and depth are about $67 \mathrm{~km}, 78 \mathrm{~km}$ and $5 \mathrm{~km}$ as shown in Fig. 5) is relatively flat, the out-of-plane reflections are limited. This implies that 2D inverse rays along MCS profiles are sufficient to image most of the bathymetry below the profiles. However, due to the sparse spacing of $10-35 \mathrm{~km}$, proper inverse-ray imaging cannot be achieved when the variation of the bathymetry exceeds $300 \mathrm{~m}$ between neighboring profiles (elliptical areas in Fig. 5b).

\subsection{SEG/EAEG Over-Thrust Model}

In quest of oil and gas embedded in the complex faults and folds, a 3D over-thrust model with eleven layers developed by SEG and EAEG has been intensively investigated for seismic modeling and inversion. In this study, we only image the fourth layer of the over-thrust model from the top (Fig. 6) since the complex faults exist in the central region of the layer. Reflected rays on the fourth layer of SEG/EAEG over-thrust model are traced along orthogonal profiles with profile spacing of $1 \mathrm{~km}$ and $4 \mathrm{~km}$, respectively, in the complex and flat regions (black dots in Fig. 7c). Along a profile, the shot interval, near offset, receiver interval and channel number are set to $1.25 \mathrm{~km}, 0.21 \mathrm{~km}, 0.1 \mathrm{~km}$ and 48 , respectively. A lot of reflection points in the central region of the thrust faults are not located along profiles since out-of-plane reflections are occurred due to the complexity of SEG/EAEG over-thrust model. 
Tan Kin Wang \& Kai Ming Chang

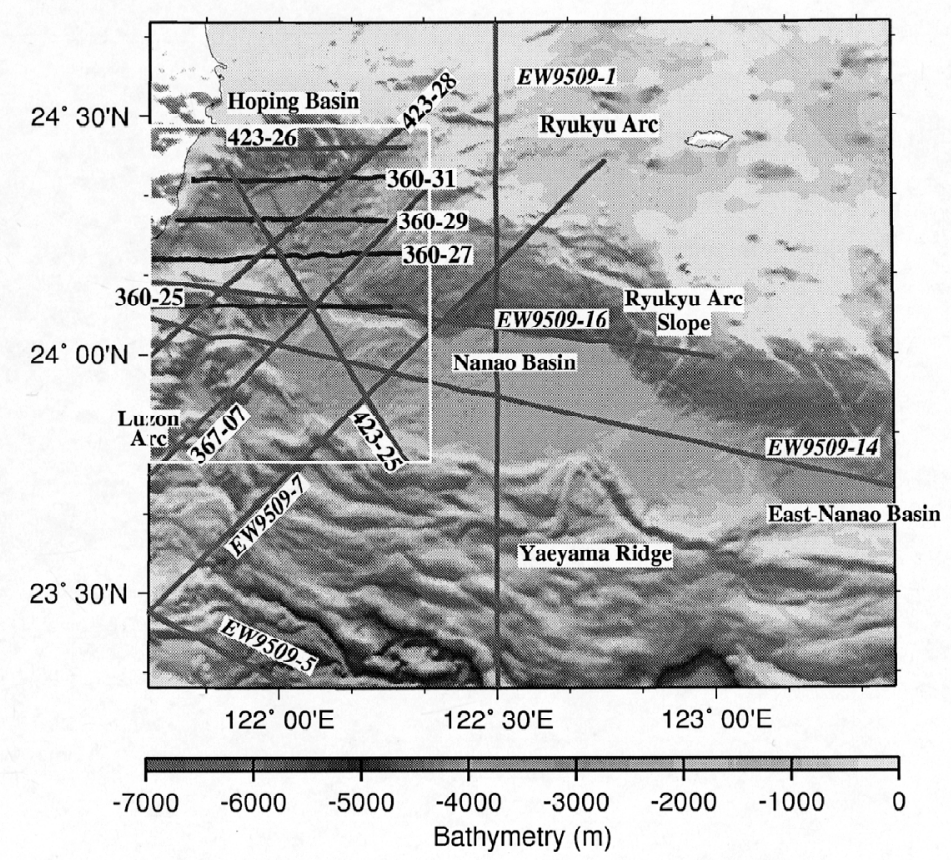

Fig. 4. Seismic lines and bathymetry (Liu et al. 1998) in the southernmost Ryukyu subduction zone. White frame indicates the study area of the Hoping Basin.

Fig. 5. (a) Tracing of reflected rays based on seven MCS profiles in the Hoping Basin and (b) inverse-ray imaging of the basin bathymetry. Insufficient inverse-rays are shown inside the elliptical areas of (b). 


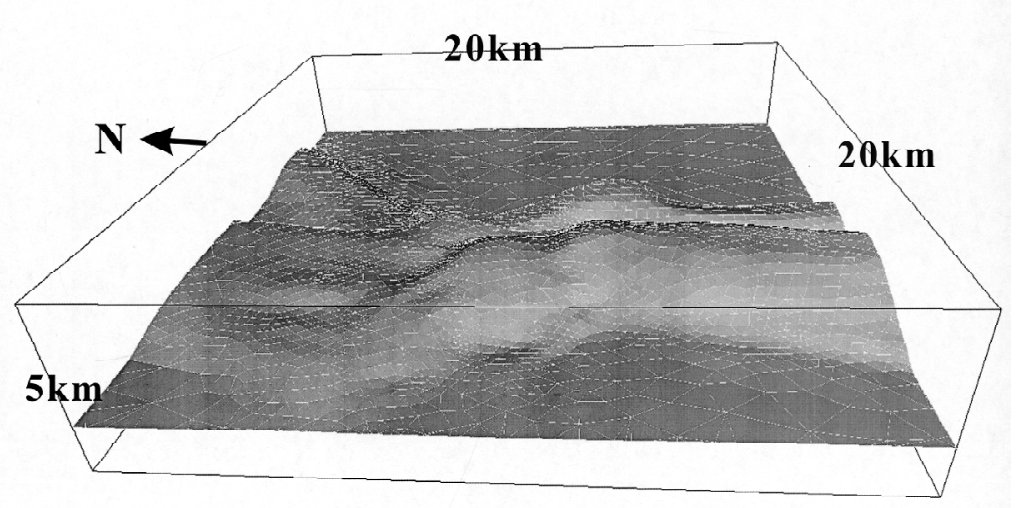

Fig. 6. The fourth layer of the SEG/EAEG over-thrust model.

Reflected interfaces of the over-thrust model (Fig. 7a) are imaged by incorporating traveltime sets across profiles. However, due to incorrect selection of travel-time sets across profiles in the complex structure of the over-thrust model, the depth errors of inverse-ray imaging are greater than $0.2 \mathrm{~km}$ in the central fault zone (red crosses and circles in Fig. 7b). These large errors can be readily identified and thus can be disregarded when the imaging density is less than 200 points $\mathrm{km}^{-2}$ (Fig. 7c) and/or the uncertainty of the imaging depth is greater than $0.2 \mathrm{~km}$ (Fig. 7d). Although inverse-ray imaging based on a 2D acquisition is insufficient to determine the whole interface of the model, it provides a general trend of the interface depth for which 3D imaging from other techniques is difficult based on the 2D data set. If the profile spacing is a few times denser than the current spacing, the full coverage and the accurate depth of the imaging interfaces can be expected.

\section{CONCLUSION}

Inverse-ray imaging of a planar reflector is presented based on reflected travel-times of common-shot gathers along two oblique profiles. We first assume all travel-times resulting from the same planar reflector, and then use any two travel-time sets along two profiles to determine the reflector normal. Several criteria based on the shortest distance between the source and the reflector, the same normal vector of the reflector, and the same ray distance are subsequently considered to delete incorrect results.

Applications of this method to a relatively flat basin and a complex over-thrust demonstrate the capability of inverse rays for imaging pseudo-3D reflectors between sparse profiles. Once travel times have been recorded along at least two profiles within a Fresnel zone, this method can resolve the structural interfaces for which 3D imaging from other techniques is difficult based on 2D data sets. Therefore, the inverse-ray method developed in this paper can image a pseudo-3D structure even when profile spacing $(>1 \mathrm{~km})$ is far beyond the grid spac- 
Tan Kin Wang \& Kai Ming Chang

(a)

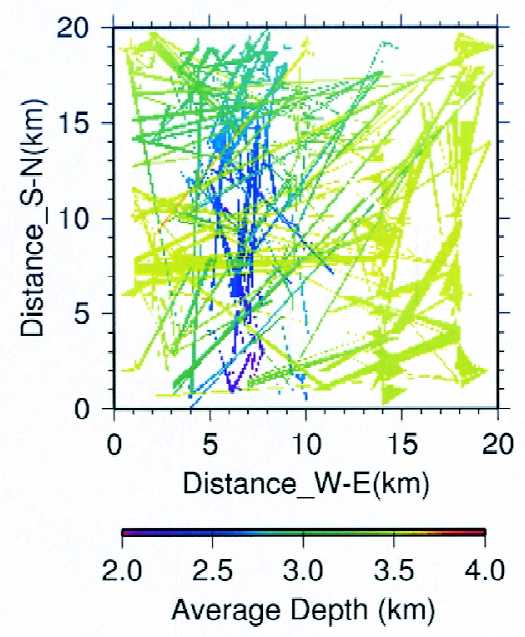

(c)
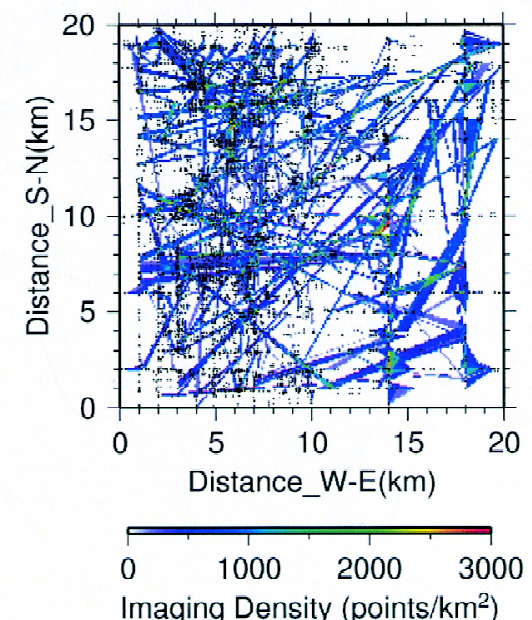

(b)

Depth Error (km)

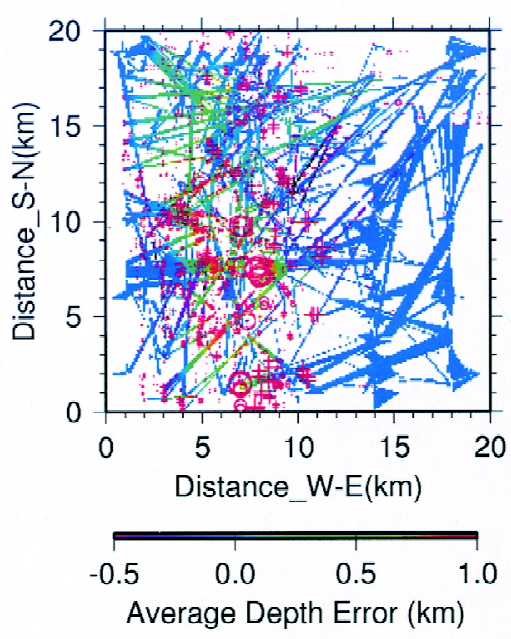

(d)

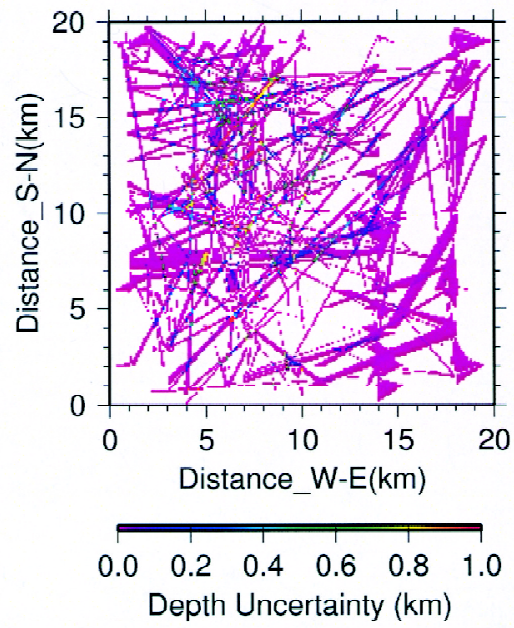

Fig. 7. (a) Average depth, (b) average depth error, (c) imaging density, and (d) depth uncertainty of the fourth layer of the SEG/EAEG over-thrust model imaged by inverse reflected rays. Grid interval of color images in (a)-(d) is $0.1 \mathrm{~km}$. Red crosses and circles in (b) denote the imaging points with a depth more than $0.2 \mathrm{~km}$ shallower and deeper than the true depth, respectively. Black dots in (c) are reflection points of inverse rays. 
ing required in the classical approaches such as migration and tomography.

Although the prestack inverse rays presented in this paper are less practical due to their limitations in determining of a homogeneous layer, they will be extended to image 3D inhomogeneous multi-layered media. For example, sedimentary and crustal structures can be investigated based on common-shot gathers from MCS acquisition and the common-receiver gathers from ocean-bottom seismometers (OBS) survey. The existing MCS/OBS profiles off eastern Taiwan provide good data sets for imaging seismogenic structures and for evaluating earthquake hazard in the southernmost Ryukyu subduction zone.

Acknowledgements We would like to thank Professor Chien-Ying Wang for providing us valuable suggestions to improve this paper. This research was supported by National Science Council, R.O.C. through the grant NSC 91-2611-M-019-006 and NSC 92-2611-M-019-015.

\section{REFERENCES}

Billette, F., and G. Lambare, 1998: Velocity macro-model estimation from seismic reflection data by stereotomography. Geophys. J. Int., 135, 671-690.

Billette, F., S. L. Begat, P. Podvin, and G. Lambare, 2003: Practical aspects and applications of 2D stereotomography. Geophys., 68, 1008-1021.

Chang, K. M., 2004: Imaging of a 3D homogeneous layer through multi-channel reflected rays, M. S. Thesis, Inst. App. Geophys., Nat. Taiwan Ocean Univ., 29.

Donihoo, K., N. Bernitsas, N. Dai, G. Martin, and D. Shope, 2001: Is depth imaging a commodity? The impact of new imaging technologies and web-based collaboration. The Leading Edge, 20, 486-496.

Gjoystdal, H., and B. Ursin, 1981: Inversion of reflection times in three dimensions.Geophysics, 46, 972-983.

Gray, S. H., J. Etgen, J. Dellinger, and D. Whitmore, 2001: Seismic migration problems and solutions. Geophys., 66, 1622-1640.

Hua, B. L., and G. A. McMechan, 2003: Parsimonious 2-D prestack Kirchhoff depth migration. Geophys., 68, 1043-1051.

Kleyn, A. H., 1977: On the migration of reflection time contour maps. Geophys. Prosp., 25, 125-140.

Lallemand, S., C. S. Liu, and Y. Font, 1997: A tear fault between the Taiwan orogen and the Ryukyu subduction zone. Tectonophys., 274, 171-190.

Levin F. K., 1971: Apparent velocity from dipping interface reflections. Geophysics, 36, 510516.

Liu, C. S., S. Y. Liu, S. E. Lallemand, N. Lundberg, and D. L. Reed, 1998: Digital elevation model offshore Taiwan and its tectonic implications. Terr. Atmos. Ocean. Sci., 9, 705738.

Maber, S. M., and D. M. Hadley, 1986: Stable and accurate depth determination using map migration. Geophys., 51, 1319-1320. 


\section{Tan Kin Wang \& Kai Ming Chang}

May, B. T., and J. D. Covey, 1981: An inverse ray method for computing geologic structures from seismic reflections - Zero-offset case. Geophys., 46, 268-287.

Operto, M. S., S. Xu, and G. Lambare, 2000: Can we quantitatively image complex structures with rays? Geophys., 65, 1223-1238.

Pivot, F., 1997: A geometrical method for migrating 3-D or 2.5-D seismic reflection data. Geophys. Res. Lett., 24, 3321-3324.

Reilly, J. M., 1991: Integrated interpretation, 3D map migration and VSP modeling project, Northern U. K. Southern gas basin. Geophys. Prosp., 39, 253-278.

Sattlegger, J. W., 1969: Three-dimensional seismic depth computation using space-sampled velocity logs. Geophys., 34, 7-20.

Wang, T. K., 2002: Prestack inverse-ray imaging of a 2D homogeneous layer: a tutorial study. Terr. Atmos. Ocean. Sci., 13, 399-416.

Wang, T. K., 2005a: Inverse-ray imaging from triangulation of zero-offset reflection times. Geophys. J. Int., accepted.

Wang, T. K., S. F. Lin, C. S. Liu, and C. S. Wang, 2004: Crustal structure of the southernmost Ryukyu subduction zone: OBS, MCS and gravity modeling. Geophys. J. Int., 157, 147163.

Wang, T. K., 2005b: The stability of 2-D multi-layered structures imaged by inversion of reflected rays. Geophys., accepted.

\section{APPENDIX A. Unit Normal Vector of a Reflector}

Let the shooting direction of a MCS profile be set as $X_{i}$ axis and the shortest distance between a source $\left(S_{i}\right)$ and a planar reflector is $h_{i}$ (Fig. 1). Then a vector $\left(\overrightarrow{S_{i} S_{i}^{*}}\right)$ along the shortest distance $\left(h_{i}\right)$ is parallel to the unit normal vector $(\hat{\mathbf{N}})$ of the reflector, for which:

$$
\begin{aligned}
\hat{\mathbf{N}} & =\sin \phi \cos \theta \hat{X}+\sin \phi \sin \theta \hat{Y}+\cos \phi \hat{Z} \\
& =\sin \phi \cos \theta_{i} \hat{X}_{i}+\sin \phi \sin \theta_{i} \hat{Y}_{i}+\cos \phi \hat{Z}_{i},
\end{aligned}
$$

where $\phi$ is the true dip, $\theta$ and $\theta_{i}$ is angles of the dip line $\left(\overrightarrow{S_{i} S^{\prime \prime}}\right.$ in Fig. A1) of the reflector with respect to the $X$ and $X_{i}$ axes, respectively. However, neither the true dip nor the dip line in equation (A1) is known except that the direction cosine of $\hat{\mathbf{N}}$ with respect to the $X_{i}$ axis (Levin 1971)

$$
\cos \alpha_{i}=\sin \phi \cos \theta_{i},
$$

can be determined from the least-square travel-time fitting of a common-shot gather along a profile (Wang 2002).

Two direction $\operatorname{cosines~}\left(\cos \alpha_{1}\right.$ and $\cos \alpha_{2}$ ) along two profiles ( $X_{1}$ and $X_{2}$ axes) can be used for determining the true dip and the dip line of the reflector in the following. Since the 
angles of the dip line ( $\theta$ and $\theta_{i}$ ) with respect to the $X$ and $X_{i}$ axes are related to each other by considering the profile azimuth $\left(\theta_{i}^{*}\right)$ as shown in Fig. A1, we observe that:

$$
\theta_{i}=\theta-\theta_{i}^{*}, i=1,2 \text {. }
$$

To substitute the equation (A3) into equation (A2), we obtain

$$
\left[\begin{array}{c}
\sin \phi \cos \theta \\
\sin \phi \sin \theta
\end{array}\right]=\frac{1}{\sin \left(\theta_{2}^{*}-\theta_{1}^{*}\right)}\left[\begin{array}{cc}
\sin \theta_{2}^{*} & -\sin \theta_{1}^{*} \\
-\cos \theta_{2}^{*} & \cos \theta_{1}^{*}
\end{array}\right]\left[\begin{array}{c}
\cos \alpha_{1} \\
\cos \alpha_{2}
\end{array}\right] .
$$

The denominator in equation (A4) should not be zero except when $\alpha_{1}=\alpha_{2}=\pi / 2$. Therefore, two profiles used for determining the reflector normal should not be parallel to each other. Equations (A1) and (A4) also indicate that $X$ and $Y$ components of the unit normal vector of the reflector can be determined from equation (A4) whereas the $Z$ component of $\hat{\mathbf{N}}$ can be calculated from $N_{z}=\sqrt{1-N_{x}^{2}-N_{y}^{2}}$.

The only exception of using parallel profiles $\left[\sin \left(\theta_{2}^{*}-\theta_{1}^{*}\right)=0\right]$ is when their apparent dips are zero $\left(\alpha_{i}=\pi / 2\right)$ so that equation (A4) is no longer applicable. For determining the unit normal vector of a reflector in this special case (Fig. A2), a unit vector perpendicular to the profiles is set:

$$
\hat{X}_{\perp}=\sin \theta_{i}^{*} \hat{e}_{x}-\cos \theta_{i}^{*} \hat{e}_{y},
$$

which is along the direction of the dip line of the reflector. By considering the right triangle $\Delta S_{i} E F$ as shown in Fig. A2, the true dip $(\phi)$ of the reflector can be determined from:

$$
\sin \phi=\frac{h_{i}-h_{j}}{\overrightarrow{S_{i} S_{j}} \cdot \hat{X}_{\perp}} .
$$

Therefore, when the apparent dips along two parallel profiles are zero, the unit normal vector of the reflector is:

$$
\hat{\mathbf{N}}=\sin \phi \hat{X}_{\perp}+\cos \phi \hat{e}_{z} .
$$


Tan Kin Wang \& Kai Ming Chang

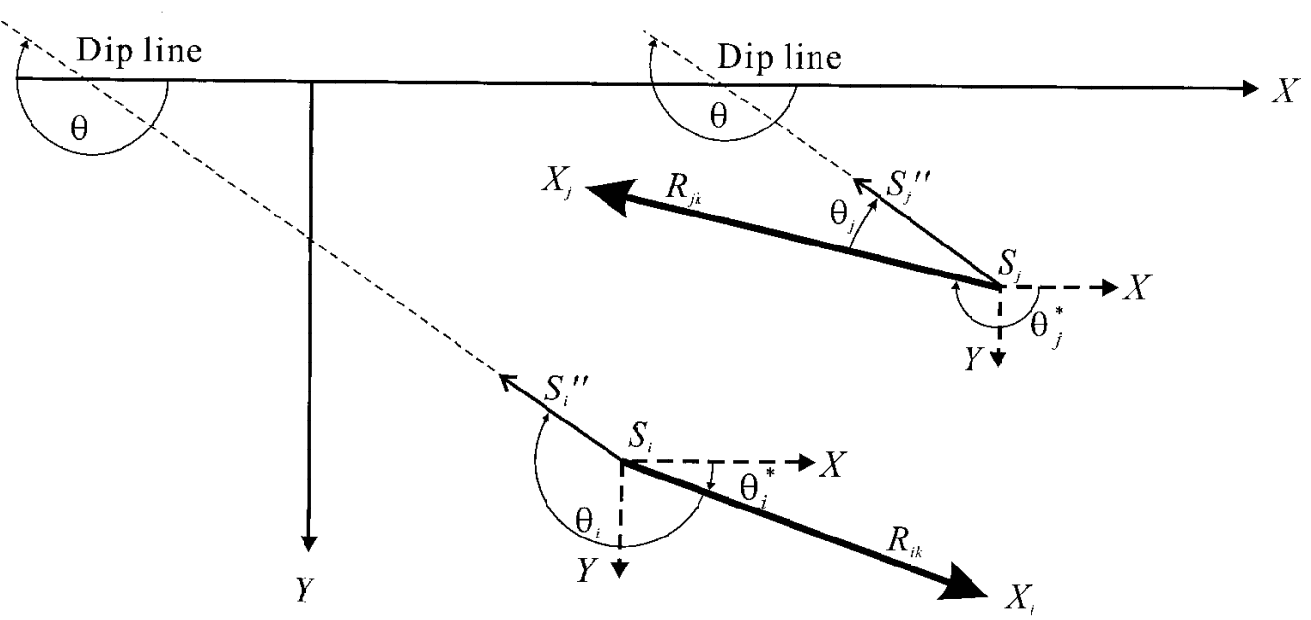

Fig. A1. Two oblique profiles $\left(X_{i}\right.$ and $X_{j}$ ) and the dip line of a planar reflector projected onto the surface. Azimuths of the profile $X_{i}$ (or $X_{j}$ ) and the dip line are denoted by $\theta_{i}^{*}$ (or $\theta_{j}^{*}$ ) and $\theta$, respectively. $\theta_{i}$ (or $\theta_{j}$ ) is the angle between the dip line and the profile $X_{i}\left(\right.$ or $\left.X_{j}\right)$.

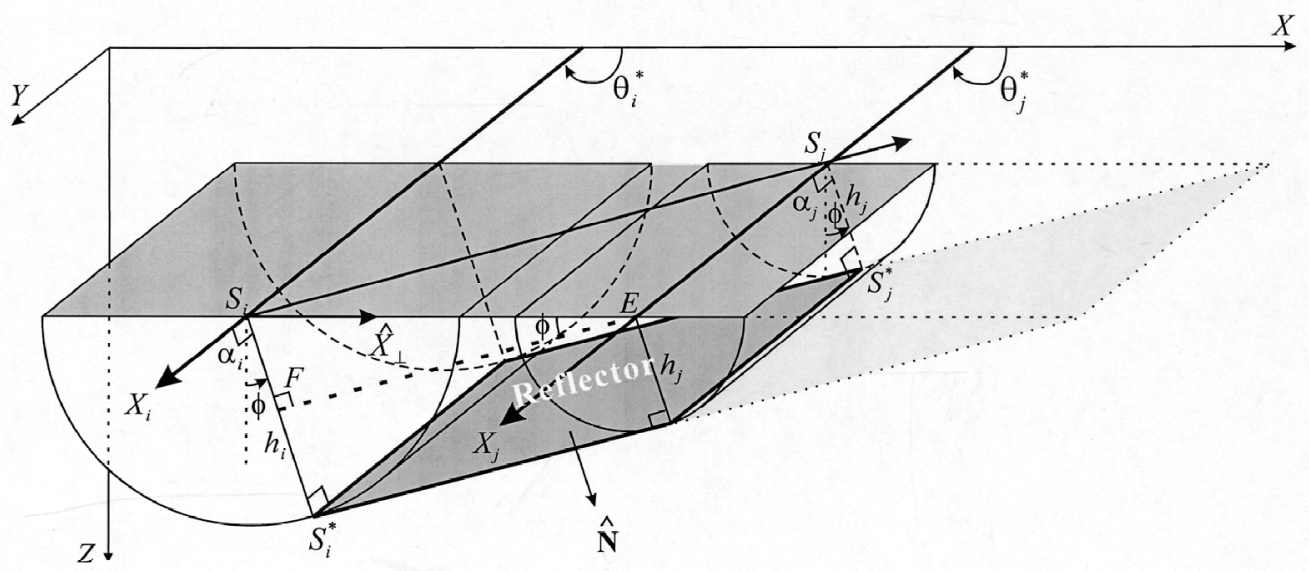

Fig. A2. A planar reflector imaged from a tangent plane of two circularly cylindrical envelopes when $\alpha_{i}, \alpha_{j}=\pi / 2$ along two parallel profiles. $\hat{X}_{\perp}$ is a unit vector perpendicular to the profiles. 